Document downloaded from:

http://hdl.handle.net/10251/57332

This paper must be cited as:

Escalante Fernández, JM.; Martínez Abietar, AJ. (2012). Theoretical study about the gain in indirect bandgap semiconductor optical cavities. Physica B: Condensed Matter. 407(12):2044-2049. doi:10.1016/j.physb.2012.02.002.

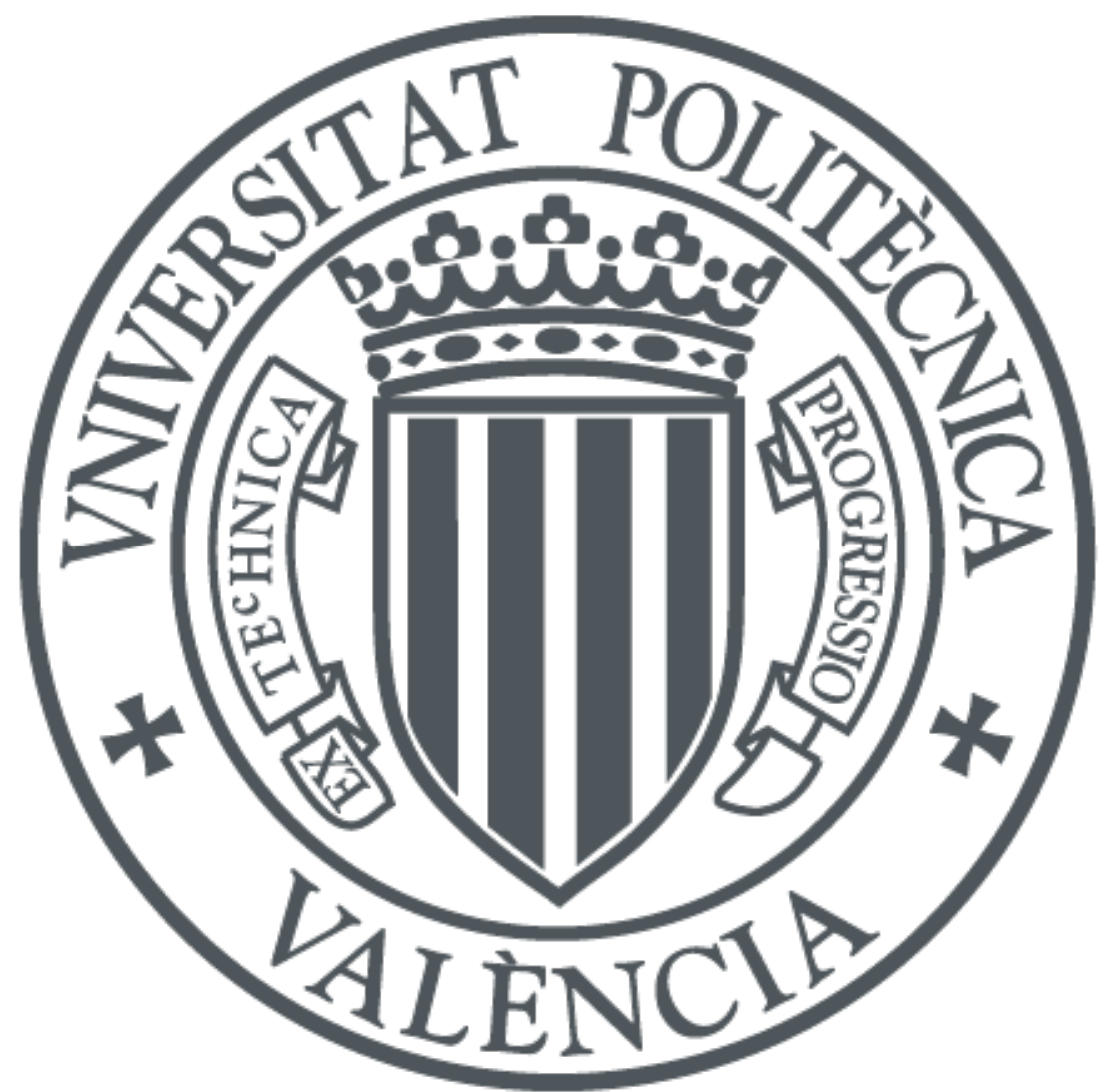

The final publication is available at

http://dx.doi.org/10.1016/j.physb.2012.02.002

Copyright Elsevier

Additional Information 


\title{
Theoretical study about the gain in indirect bandgap semiconductor optical cavities
}

\author{
Jose M. Escalante* and Alejandro Martínez \\ Nanophotonics Technology Center, Universidad Politécnica de Valencia, 46022 \\ Valencia, Spain \\ *Electronic mail: jmescala@ntc.upv.es
}

\begin{abstract}
Indirect bandgap semiconductors such as silicon are not efficient light emitters because a phonon with a high momentum is required to transfer an electron from the conduction to the valence band. In a recent study [M. J. Chen et al., Japanese Journal of Applied Physics 45, 6576-6588 (2006)] an analytical expression of the optical gain in bulk indirect bandgap semiconductors was obtained. The main conclusion was that the free-carrier absorption was much higher than the optical gain at ambient temperature, which prevents lasing. In this work, we consider the case in which the semiconductor material is engineered to form an optical cavity characterized by a certain Purcell factor. We obtain that although the optical gain is increased, losses due to free carriers increase in the same way so lasing is also prevented even when creating a high- $Q$ optical cavity.
\end{abstract}




\section{Introduction}

Silicon photonics has boomed in the last few years as a promising way to create lowcost, high-speed optical interconnects that replace copper wires in future computers [13]. The main advantage of using silicon as a photonic material is that it can be processed in microelectronics foundries with high yield and low cost. However, silicon has a main drawback: it is an indirect bandgap semiconductor in which radiative transitions are unlikely and, as so, a very inefficient light emitter. A silicon laser would allow monolithic integration of photonics and electronics on a same chip [2]. Despite of huge research efforts by many groups around the world, an electrically-pumped roomtemperature silicon laser - perhaps the most pursued challenge within photonics remains elusive.

Bulk crystalline silicon has an indirect energy bandgap so emission of light requires the participation of phonons with the right momentum in order to satisfy the momentum conservation. The low probability of the phonon-mediated radiative recombination process makes silicon a highly inefficient light source. In fact, there exists the general belief that optical gain and thus laser operation in indirect bandgap semiconductors is not possible because the small optical gain- which could be achieved in principle via band-band transitions mediated by phonons- will always be overcompensated by free carrier absorption, regardless of the excitation conditions [4]. This statement, together with the fact that no silicon lasing at room temperature has been reported yet, explains why typically III-V semiconductors having a direct band gap has been used to implement lasers in the near -infrared regime (such as the important optical communications band at wavelength about $1550 \mathrm{~nm}$ ).

However, some recent theoretical works analysing the possibility of achieving optical gain in indirect bandgap semiconductors at room temperature have given rise to certain controversy. For instance, Trupke and co-workers suggested that optical gain in silicon is theoretically possible and pointed out that the most suitable energy region is the subbandgap region (near infrared) where processes involving phonons could help in achieving gain [5]. Moreover, they obtained that indirect optical transitions can provide negative absorption, i.e., optical gain without an electronic population inversion, but with the assistance of proper phonons. These theoretical arguments were also supported in Ref. [6] where an analytical expression for optical gain via phononassisted optical transitions in indirect bandgap semiconductors is presented. The magnitude of optical gain in bulk crystalline silicon is calculated and shown to be smaller than the free carrier absorption at room temperature. However, it is shown, for 
the first time, that the optical gain is greater than the free carrier absorption in bulk crystalline silicon at the temperature below $23 \mathrm{~K}$ [6].

Other some experimental works have reported an increased photoluminescence from silicon when photonic cavities with high Q-factor are created Ref.[7-15]. In this case, the generation of photons is enhanced in comparison to the case of bulk silicon [16] because of the Purcell effect [17] (or, in other works, the increase of the optical density of states inside the cavity). However, those results have been mainly attributed to an increase of the spontaneous emission rate but nor lasing neither optical gain have been directly observed. So the natural question that arises is: can optical gain at room temperature be obtained in indirect bandgap semiconductors when an optical cavity instead of a bulk material is considered? In this work we try to answer this question by starting from the analytical results obtained in Ref. [6].

\section{Rate equations}

Figure 1 shows a schematic diagram that describes all possible optical transitions taking place in an indirect bandgap semiconductor such as silicon. It can be seen how three different kinds of particles are involved in this process: electrons, photons and phonons (which are not involved in the same process when taking place in direct bandgap semiconductors).

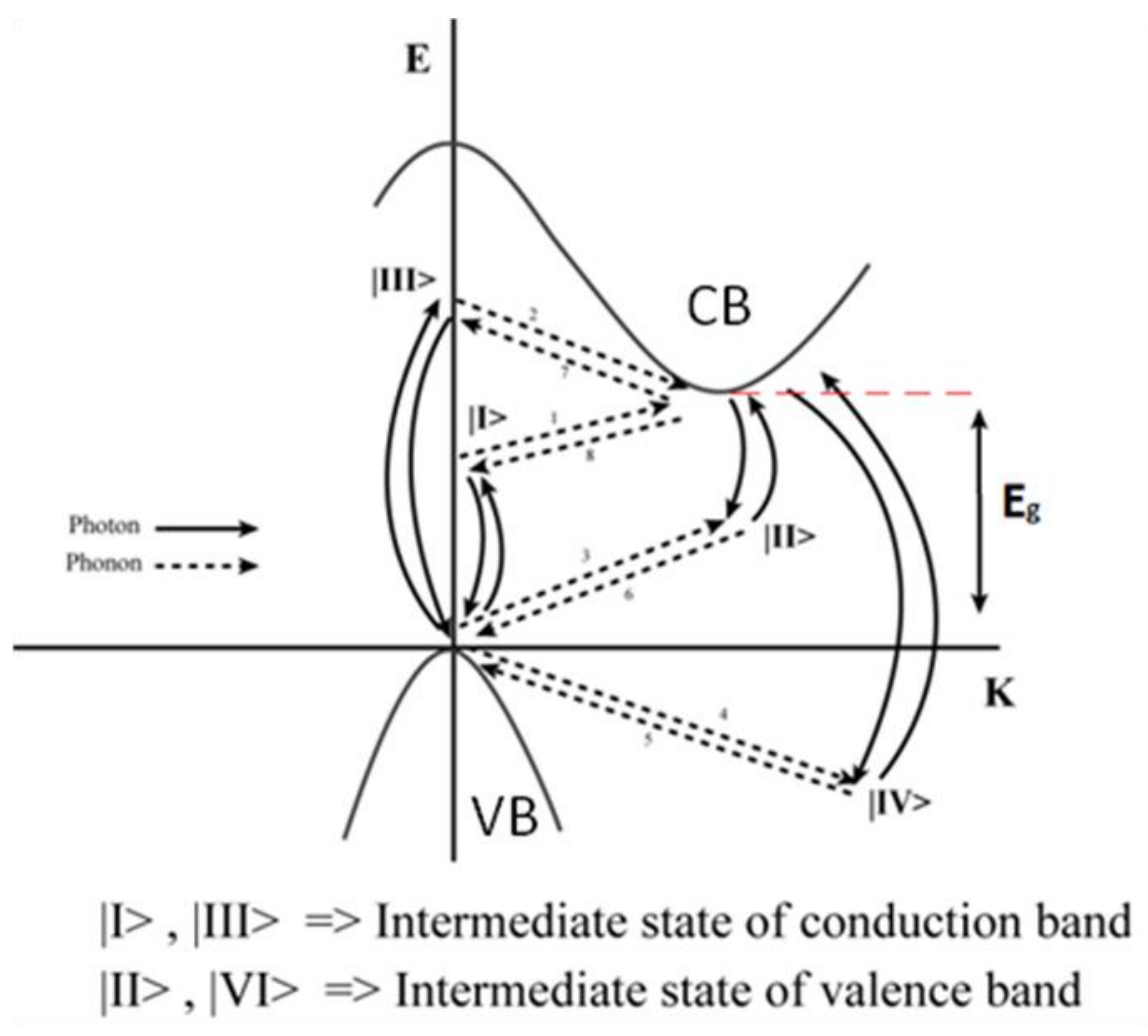

Figure1. Schematic diagram of all possible optical transitions in an indirect bandgap semiconductor 
In Ref. [6], M. J. Chen and co-workers obtained a theoretical expression for the different transition rates that occur in bulk indirect bandgap semiconductors. For the sake of clarity, we represent here the expression of these rates:

$$
\begin{aligned}
& R_{s p}=M\left(n_{q}+1\right) \cdot N P \\
& R_{s t}=M \cdot n_{p}\left(n_{q}+1\right) \cdot N P \\
& R_{a b}=M \cdot n_{p} n_{q} \cdot N P \exp \left(\frac{(\hbar \omega+\hbar \Omega-\Delta F)}{K_{B} T}\right) \\
& M=\frac{\pi}{8} B_{s p}\left(\hbar \omega+\hbar \Omega-E_{g}\right)^{2} \exp \left(-\frac{\left(\hbar \omega+\hbar \Omega-E_{g}\right)}{K_{B} T}\right)
\end{aligned}
$$

In Eqs. (1)-(4), $R_{\mathrm{sp}}$ is the spontaneous emission rate, $R_{\mathrm{st}}$ is the stimulated emission rate, $R_{\mathrm{st}}$ is the absorption rate, $n_{\mathrm{p}}$ is the photon occupation number, $n_{\mathrm{q}}$ is the phonon occupation number, $\hbar \omega$ is the photon energy, $\hbar \Omega$ is the phonon energy, $\Delta F$ is the difference between the quasi-Fermi levels for electrons and holes, $N$ is the electron concentration, $P$ is hole concentration (in our study we consider that $N=P$ ), $E_{g}$ is the indirect bandgap energy, $K_{\mathrm{B}}$ is the Boltzman constant and $T$ is the temperature (we assume room temperature throughout this work). In this work we consider silicon as indirect bandgap semiconductor, so the radiative transition rates can be calculated using the Eqs.(1)-(4) and the values given in the Table I in Ref.[6]. We also consider all the assumptions made in Ref. [6].

The following equations system ${ }^{1}$ [6] governs the temporal variation of the photon density $\left(N_{\mathrm{p}}\right)$, the phonon density $\left(N_{\mathrm{q}}\right)$ and the carrier density $(N)$ :

$$
\begin{aligned}
& \frac{d N}{d t}=R_{p}-R_{s t, B}(\hbar \omega)+R_{a b, B}(\hbar \omega)-R_{s p, B}(\hbar \omega)-\frac{N}{\tau_{c}} \\
& \frac{d N_{p}}{d t}=R_{s t, B}(\hbar \omega)-R_{a b, B}(\hbar \omega)+\beta R_{s p, B}(\hbar \omega)-\frac{N_{p}}{\tau_{p}} \\
& \frac{d N_{q}}{d t}=R_{s t, B}(\hbar \omega)-R_{a b, B}(\hbar \omega)+R_{s p, B}(\hbar \omega)-\frac{N_{q}-N_{q 0}}{\tau_{q}}
\end{aligned}
$$

where $R p$ is the pumping rate by current injection or optical excitation, $\beta$ is the spontaneous emission factor representing the fraction of spontaneous emission

\footnotetext{
${ }^{1}$ The subscript B stands for the different rates in bulk silicon.
} 
entering the optical mode has been considered, $N_{q o}$ is the phonon density at thermodynamic equilibrium, and $\tau_{c}, \tau_{p}$ and $\tau_{q}$ are the lifetime of carriers, photons and phonons, respectively. The losses of photons due to the effects such as optical scattering or free carrier absorption can be characterized by a photon lifetime $\tau_{p}$ [6]. The loss of phonons (last term of Eq.(5c), which represent the anharmonic phonon interaction, can be characterized by a phonon lifetime $\tau_{\mathrm{q}}[6]$. The recombination lifetime of carriers is given by $1 / \tau_{C}=1 / \tau_{C, R A D}+1 / \tau_{C, N R A D}=1 / \tau_{C, R A D}+1 / \tau_{C, S R H}+1 / \tau_{C, \text { Auger }}$. In Ref.[6], it is assumed that the non-radiative recombination rate is determined by the non-radiative Shockley-Read-Hall (SRH) mechanism. However, in the case of a very high carrier density in silicon, the Auger recombination lifetime is the dominant recombination mechanism, so $1 / \tau_{\mathrm{C}, \mathrm{SRH}}<<1 / \tau_{\mathrm{C}, \text { Auger }},[18-20]$. Considering the above and taking into account the carrier density that we consider in this work $\left(\sim 10^{19} \mathrm{~cm}^{-3}\right)$ then we get $\tau_{\mathrm{C}, \mathrm{RAD}}=10^{-4}$ and $\tau_{\mathrm{C}, \mathrm{NRAD}}=10^{-7}$ in silicon bulk.

\section{Increase of the optical gain with Purcell factor.}

The system of equations (5) was solved in [6] for bulk silicon. In this work we have solved the same system but considering a photonic cavity characterized by a quality factor $(Q)$, a modal volume $\left(V_{0}\right)$ and Purcell factor $\left(F_{\mathrm{p}}\right)$. These three parameters are related to each other by the following equation [17]:

$$
F_{P}=\frac{3 Q(\lambda / n)^{3}}{4 \pi^{2} V_{0}},
$$

where $\lambda$ is the resonant wavelength of the cavity and $n$ its refractive index. It has to be mentioned that we consider that the cavity only affects the photonic density of states by means of $F_{P}$ but it has no effect on the statistics of the phonons involved in the emission process. This is a good assumption taking into account that the wavelength of the phonons involved in the emission process is much smaller than the optical cavity size (which should be at least half a photon wavelength) so that phonons see a bulk material.

In the system under consideration, the spontaneous emission $\left(B_{\mathrm{sp}}\right)$, stimulated emission $\left(B_{\mathrm{st}}\right)$ and absorption $\left(B_{\mathrm{ab}}\right)$ coefficients are given by the Fermi Golden Rule, $W_{i \rightarrow f}=\frac{2 \pi}{\hbar}|\langle f|H| i\rangle|^{2} \rho$. For instance, the spontaneous emission coefficient can be obtained as:

$$
B_{s p}=\frac{2 \pi}{\hbar}|\langle f|H| i\rangle|^{2} \rho
$$


The Purcell factor $F_{p}$ can be obtained as the ratio between the transition rate coefficients inside the cavity $\left(\mathrm{W}_{\text {cav }}\right)$ and in bulk $\left(\mathrm{W}_{\mathrm{B}}\right)$ :

$$
F_{P}=\frac{W_{c a v}}{W_{B}}=\frac{B_{s p, c a v}}{B_{s p, B}}=\frac{\frac{2 \pi}{\hbar^{2}}\left|\left\langle f\left|H_{c a v}\right| i\right\rangle\right|^{2} \rho_{c a v}}{\frac{2 \pi}{\hbar^{2}}\left|\left\langle f\left|H_{B}\right| i\right\rangle\right|^{2} \rho_{B}}
$$

So the spontaneous emission coefficient in the cavity is obtained as:

$$
B_{s p, c a v}=F_{P} B_{s p, B}
$$

This means that the photonic cavity increases the spontaneous emission coefficient by a $F_{\mathrm{p}}$ factor. The creation of the cavity also affects several parameters by means of $F_{\mathrm{p}}$. For instance, the $M$ parameter given by Eq. (4), which is proportional to $B_{\mathrm{sp}}$, will be also proportional to $F_{\mathrm{P}}$, when considering the photonic cavity:

$$
M_{c a v}=F_{P} M_{B}
$$

The photon lifetime inside the cavity is given by [21]:

$$
\tau_{\mathrm{p}}=\frac{\lambda Q}{2 \pi c}
$$

Then, from Eq. (6) we get that the photon lifetime inside the cavity is also enhanced by $F_{\mathrm{p}}$ in comparison with the photon lifetime in bulk:

$$
\tau_{\mathrm{p}, \mathrm{cav}}=F_{P} \tau_{\mathrm{p}, \mathrm{B}}
$$

The density of states per energy interval for the single photon is [22]:

$$
D(E)=2 V n^{2} n_{g} \frac{(\hbar \omega)^{2}}{\hbar^{2} c^{3}}
$$

The group index $n_{\mathrm{g}}$ is proportional of a photonic cavity is proportional to the photon lifetime and therefore, to the quality factor, so we also get that the density of states per energy interval is enhanced by $F_{\mathrm{p}}$ when the photonic cavity is created:

$$
D(E)_{c a v}=F_{P} D(E)_{B}
$$

This is a quite intuitive result: the density of states inside the optical cavity is increased proportional to the Purcell factor. If we consider the density of states, $K_{\mathrm{p}}$, which can be calculated as $\mathrm{K}_{\mathrm{p}}=\int D(E) d E$ we get that it is also proportional to $F_{\mathrm{p}}$, as:

$$
K_{p, c a v}=F_{P} K_{p, B}
$$


It can be seen that the main effect of the optical cavity is to enhance all these parameters $\left(B_{\mathrm{sp}}, M, \tau_{\mathrm{p}}, D\right)$ by the Purcell factor.

Beside, we have to consider that $\tau_{C, R A D}=1 / W_{R A D}$, where $W_{R A D}$ is the radiative transition coefficient that is proportional to Purcell Factor. Some experimental values of the Purcell factor in light-emitting silicon optical cavities tuned close to the emission wavelength can be seen in Table I of Ref. [13]. Values between 160-1000 are reported depending strongly on the considered cavity mode. However, researchers have designed cavities with quality factors of the order of $10^{6}$ and modal volumes of the order $\sim 0.1(\lambda / n)^{3}$ at wavelengths around $1000 \mathrm{~nm}$, which would enable Purcell factor values greater than $10^{5}[23,25]$. If we consider this value of the Purcell factor we can consider, as a first approximation, that $1 / \tau_{\mathrm{C}, \mathrm{RAD}>}>1 / \tau_{\mathrm{C}, \mathrm{NRAD}}$ and then $\tau_{\mathrm{C}} \approx \tau_{\mathrm{C}, \mathrm{RAD}}$ can be considered a good approximation because the optical cavity will enhance the radiative transition in comparison with non-radiative transition. So we get:

$$
\tau_{\mathrm{C}, \mathrm{cav}}=\frac{\tau_{\mathrm{C}, \mathrm{B}}}{F_{P}}
$$

Therefore, in contrast to the previously addressed parameters, now we obtain that the total lifetime of carriers is inversely proportional to the Purcell factor when the optical cavity is created. Substituting the expressions (11), (13),(15) and (16) in our system of coupled equations (5) we get:

$$
\begin{aligned}
& \frac{d N}{d t}=R_{p}-F_{P} M_{B} n_{p}\left[\left(n_{q}+1\right)-n_{q} \exp \left(\frac{(\hbar \omega+\hbar \Omega-\Delta F)}{K_{B} T}\right)\right] N^{2}+F_{P} M_{B}\left(n_{q}+1\right) N^{2}-F_{P} \frac{N}{\tau_{c, B}} \\
& \frac{d n_{p}}{d t}=\frac{M_{B}}{K_{p, B}} n_{p}\left[\left(n_{q}+1\right)-n_{q} \exp \left(\frac{(\hbar \omega+\hbar \Omega-\Delta F)}{K_{B} T}\right)\right] N^{2}+\frac{M_{B}}{K_{p, B}}\left(n_{q}+1\right) N^{2}-\frac{n_{p}}{F_{P} \tau_{p . B}} \\
& \frac{d n_{q}}{d t}=F_{P} \frac{M_{B}}{K_{q}} n_{p}\left[\left(n_{q}+1\right)-n_{q} \exp \left(\frac{(\hbar \omega+\hbar \Omega-\Delta F)}{K_{B} T}\right)\right] N^{2}+F_{P} \frac{M_{B}}{K_{q}}\left(n_{q}+1\right) N^{2}-\left(\frac{n_{q}-n_{q 0}}{\tau_{q}}\right)
\end{aligned}
$$

In the steady-state regime all the time derivatives are zero so from Eq. (18b) we obtain:

$$
n_{p}=\frac{\frac{M_{B}}{K_{p, B}}\left(n_{q}+1\right) N^{2}}{\frac{1}{F_{P} \tau_{p . B}}-\frac{M_{B}}{K_{p, B}}\left[\left(n_{q}+1\right)-n_{q} \exp \left(\frac{(\hbar \omega+\hbar \Omega-\Delta F)}{K_{B} T}\right)\right] N^{2}}
$$

and 


$$
\frac{K_{p, B}}{\tau_{p, B}}=F_{P} M_{B}\left[\left(n_{q}+1\right)-n_{q} \exp \left(\frac{(\hbar \omega+\hbar \Omega-\Delta F)}{K_{B} T}\right)\right] N^{2}
$$

Eq. (20) stands for the threshold condition. Our results show that inside the optical cavity the threshold condition for laser oscillation is not so restrictive as in bulk and the photon loss of the resonant cavity is quickly compensated. We can also obtain the following expression for the optical gain in the cavity:

$$
g_{c a v}(\hbar \omega)=\frac{h^{3} c^{2}}{8 \pi n^{2}(\hbar \omega)^{2}} R_{s p, c a v}(\hbar \omega) \cdot\left[1-\frac{n_{q}}{n_{q}+1} \exp \left(\frac{(\hbar \omega+\hbar \Omega-\Delta F)}{K_{B} T}\right)\right]
$$

which is the same as in Ref. [6] but with the addition of the Purcell factor when considering the spontaneous emission rate:

$$
R_{s p, c a v}(\hbar \omega)=F_{P} R_{s p, B}(\hbar \omega)
$$

We can see that the optical gain increases in proportion to the Purcell factor, as it could be expected:

$$
g_{c a v}(\hbar \omega)=F_{P} g_{B}(\hbar \omega)
$$

where $g_{\mathrm{B}}$ is the optical gain in bulk and $g_{\mathrm{cav}}$ is the optical gain inside the cavity.

\section{Variation of the optical gain, photon density, phonon density, carrier density, oscillation laser threshold and threshold pumping}

As in Ref. [6], we will discuss the steady-state solutions in two different situations: below and above threshold, but now ,in the case where we have a silicon cavity.

a) Below threshold, the photon density is low, so the net stimulated emission rate can be neglected and:

$$
F_{P} R_{s t, B}(\hbar \omega)-F_{P} R_{a b, B}(\hbar \omega)=F_{P}\left[R_{s t, B}(\hbar \omega)-R_{a b, B}(\hbar \omega)\right] \approx 0
$$

Then, the rate equations become:

$$
\begin{aligned}
& R_{p}-F_{P} M_{B}\left(n_{q}+1\right) N^{2}-F_{P} \frac{N}{\tau_{c, B}}=0 \\
& \frac{M_{B}}{K_{p, B}}\left(n_{q}+1\right) N^{2}-\frac{n_{p}}{F_{P} \tau_{p . B}}=0 \\
& F_{P} \frac{M_{B}}{K_{q}}\left(n_{q}+1\right) N^{2}-\left(\frac{n_{q}-n_{q 0}}{\tau_{q}}\right)=0
\end{aligned}
$$




$$
N_{p}=K_{p} n_{p} \quad ; \quad N_{q}=K_{q} n_{q}
$$

Using the values shown in Table I of Ref. [6], which can be considered as typical values in silicon, we get that the first term in the left side of Eq. (25c) is approximately equal to $F_{\mathrm{P}}\left(n_{\mathrm{q}}+1\right) \times 10^{-4}$ whilst the second term is approximately equal to $\left(n_{q^{-}} n_{\mathrm{q} 0}\right) \times 10^{12}$. Therefore, we can neglect the first term and then approximate $n_{\mathrm{q}} \approx n_{\mathrm{q} 0}$ at room temperature, which is a good assumption provided that $F_{\mathrm{P}} \leq 10^{14}$. Then we obtain:

$$
N_{q} \approx N_{q 0}
$$

From Eq. (32a) we get that the carrier concentration is:

$$
N=\frac{-\frac{F_{P}}{\tau_{c, B}}+\frac{F_{P}}{\tau_{c, B}} \sqrt{1+4 M_{B} \frac{\tau_{c, B}^{2}}{F_{P}}\left(n_{q 0}+1\right) R_{p}}}{2 M_{B} F_{P}\left(n_{q 0}+1\right)}
$$

Using again the values of the Table I in Ref. [6] and performing some approximations we get:

$$
\begin{gathered}
N=\frac{-\frac{\mathrm{F}_{\mathrm{P}}}{\tau_{\mathrm{c}, \mathrm{B}}}+\frac{\mathrm{F}_{\mathrm{P}}}{\tau_{\mathrm{c}, \mathrm{B}}} \sqrt{1+4 M_{B} \frac{\tau_{\mathrm{c}, \mathrm{B}}^{2}}{F_{P}}\left(n_{q 0}+1\right) R_{p}}}{2 M_{B} F_{P}\left(n_{q 0}+1\right)} \\
\frac{-\frac{\mathrm{F}_{\mathrm{P}}}{\tau_{\mathrm{c}, \mathrm{B}}}+\frac{\mathrm{F}_{\mathrm{P}}}{\tau_{\mathrm{c}, \mathrm{B}}}\left(1+2 M_{B} \frac{\tau_{\mathrm{c}, \mathrm{B}}^{2}}{F_{P}}\left(n_{q 0}+1\right) R_{p}\right)}{2 M_{B} F_{P}\left(n_{q 0}+1\right)}=\frac{\tau_{\mathrm{c}, \mathrm{B}}}{F_{P}} R_{p}
\end{gathered}
$$

The higher the Purcell factor (or the Q-factor of the photonic cavity), the better the approximation in Eq. (28) will be. Finally, by substituting Eqs. (26) and (28) into Eq. (25b), the photon density in the cavity is obtained as:

$$
N_{p} \approx M_{B} \tau_{c, B}^{2} \tau_{p, B}\left(n_{q 0}+1\right) R_{p}^{2}
$$

b) Above threshold, it occurs that $\Delta F>>\hbar \omega+\hbar \Omega$, so finally the threshold condition for laser oscillation is:

$$
\frac{K_{p, B}}{\tau_{p, B}}=F_{P, B} M_{B}\left(n_{q}+1\right) N^{2}
$$

and the system of coupled equations in the steady-state is now: 


$$
\begin{aligned}
& R_{p}-F_{P} M_{B} n_{p}\left(n_{q}+1\right) N^{2}-F_{P} M_{B}\left(n_{q}+1\right) N^{2}-F_{P} \frac{N}{\tau_{c, B}}=0 \\
& \frac{M_{B}}{K_{p, B}} n_{p}\left(n_{q}+1\right) N^{2}+\frac{M_{B}}{K_{p, B}}\left(n_{q}+1\right) N^{2}-\frac{n_{p}}{F_{P} \tau_{p . B}}=0 \\
& F_{P} \frac{M_{B}}{K_{q}} n_{p}\left(n_{q}+1\right) N^{2}+F_{P} \frac{M_{B}}{K_{q}}\left(n_{q}+1\right) N^{2}-\left(\frac{n_{q}-n_{q 0}}{\tau_{q}}\right)=0
\end{aligned}
$$

Substituting the threshold condition given by Eq. (30) into Eqs. (31a) and (31c) the following equation is obtained

$$
n_{\mathrm{q}}^{3}+\left(1-2 \mathrm{~A}_{1}\right) n_{\mathrm{q}}^{2}+\left(\mathrm{A}_{1}^{2}-2 \mathrm{~A}_{1}\right) n_{q}+\left(\mathrm{A}_{1}^{2}-\mathrm{A}_{2}\right)=0,
$$

where

$$
\mathrm{A}_{1}=\mathrm{n}_{\mathrm{q} 0}+\frac{\tau_{\mathrm{q}} R_{p}}{K_{q}}, \quad \mathrm{~A}_{2}=\frac{K_{p, c a v} \tau_{q}^{2}}{\tau_{\mathrm{p}, \mathrm{cav}} M_{c a v} \tau_{c, c a v}^{2} K_{q}^{2}}
$$

Using the values in Ref. [6] again, we get:

$$
\mathrm{A}_{2}=\frac{K_{p} \tau_{q}^{2}}{\tau_{\mathrm{p}} M \tau_{c}^{2} K_{q}^{2}}=\frac{F_{P} K_{p, B} \tau_{q}^{2}}{F_{P} \tau_{\mathrm{p}, \mathrm{B}} F_{P} M_{B} \frac{\tau_{c, B}^{2}}{F_{P}^{2}} K_{q}^{2}}=\frac{F_{P}}{10^{19}}
$$

If $F_{p}<<10^{14}$ the term $A_{1}^{2}$ is found to be much greater than $A_{2}$, so $A_{2}$ can be neglected and the approximate solution to Eq. (32) is:

$$
n_{q} \approx \mathrm{A}_{1}=\mathrm{n}_{\mathrm{q} 0}+\frac{\tau_{\mathrm{q}} R_{p}}{K_{q}}
$$

And therefore:

$$
N_{q} \approx \mathrm{N}_{\mathrm{q} 0}+\tau_{\mathrm{q}} R_{p}
$$

Substituting Eq. (35) into Eq. (30) we get the following approximation for the threshold of the carrier density:

$$
N=\sqrt{\frac{K_{p, B}}{\tau_{p, B} F_{P, B} M_{B}\left(\mathrm{n}_{\mathrm{q} 0}+1+\frac{\tau_{\mathrm{q}} R_{p}}{K_{q}}\right)}} \equiv N_{t h}
$$


We can see that $F_{\mathrm{P}}$ decreases the carrier density threshold to get the laser oscillation. Substituting Eqs. (36) and (30) into Eq. (31a) and using the approximation $n_{\mathrm{q}} \approx n_{\mathrm{q} 0}$, we get:

$$
R_{p}-n_{p} \frac{K_{p, B}}{\tau_{p, B}}-F_{P} M_{B}\left(n_{q}+1\right) N^{2}-F_{P} \frac{N}{\tau_{c, B}}=0
$$

and

$$
\begin{gathered}
N_{p}=F_{p} \tau_{p, B}\left(R_{p}-F_{P} M_{B}\left(n_{q}+1\right) N_{t h}{ }^{2}-F_{P} \frac{N_{t h}}{\tau_{c, B}}\right)=F_{p} \tau_{p, B}\left(R_{p}-R_{t h}\right) \\
R_{t h}=F_{P} M_{B}\left(n_{q}+1\right) N_{t h}{ }^{2}+F_{P} \frac{N_{t h}}{\tau_{c, B}}
\end{gathered}
$$

Where we must take into account that $n_{p} K_{p, B}$ is the photon density in bulk but $F_{P} n_{p} K_{p, B}$ is the photon density inside the photonic cavity, and $R_{\mathrm{th}}$ is the pumping rate at threshold. The final expressions we get for the carrier, photon and phonon densities are summarized in Table I.

Table I Summary of theoretical expression for for the carrier, photon and phonon densities

\begin{tabular}{|l|c|c|}
\cline { 2 - 3 } \multicolumn{1}{c|}{} & Below threshold & Above threshold \\
\hline $\begin{array}{l}\text { Carrier } \\
\text { density }\end{array}$ & $N=\frac{\tau_{c, B}}{F_{P}} R_{p}$ & $N=\sqrt{\frac{K_{p, b}}{\tau_{p, B} F_{P} M_{B}\left(\mathrm{n}_{\mathrm{q} 0}+1+\frac{\tau_{\mathrm{q}} R_{p}}{K_{q}}\right)}} \equiv \mathrm{N}_{\mathrm{th}}$ \\
\hline $\begin{array}{l}\text { Photon } \\
\text { density }\end{array}$ & $\mathrm{N}_{\mathrm{p}} \approx M_{B} \tau_{c, B}^{2} \tau_{p, B}\left(n_{q 0}+1\right) R_{p}^{2}$ & $N_{p}=F_{p} \tau_{p, B}\left(R_{p}-R_{t h}\right)$ \\
\hline $\begin{array}{l}\text { Phonon } \\
\text { density }\end{array}$ & $N_{q} \approx N_{q 0}$ & $N_{q} \approx \mathrm{N}_{\mathrm{q} 0}+\tau_{\mathrm{q}} R_{p}$ \\
\hline
\end{tabular}

Table 1 Final expressions for $N_{p}, N_{q}$ and $N$ in the photonic cavity.

\section{Numerical results}

In all the numerical results displayed in this section we employ again the parameters summarized in Table I of Ref. [6]. To start with, in Fig. 2 we represent the pumping rate 
at threshold, $R_{p, t h}$, as a function of the cavity Purcell factor. The $R_{\text {th }}$ dependence on $1 / \sqrt{F_{p}}$ is intuitive since $F_{\mathrm{P}}$ decreases the carrier lifetime, so increasing the pumping rate is necessary to get the population inversion. We should mention that we have considered that the carrier lifetime is equal to the radiative lifetime, which can be considered a good approximation in our scenario as previously discussed.

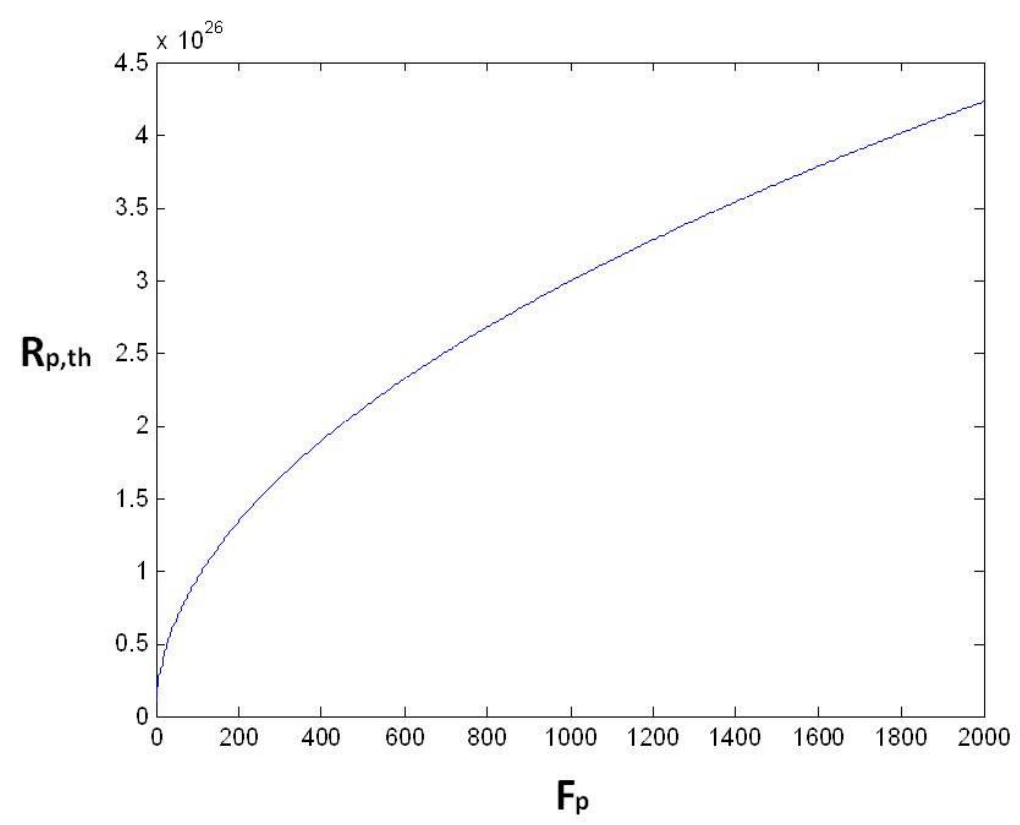

Figure 2. Dependence of $R_{p, t h}$ on the Purcell factor.

Figure 3 shows the carrier concentration, phonon density and photon density as a function of the pumping rate $R_{\mathrm{p}}$ for $F_{\mathrm{p}}$ values between 100 and 2000 (the arrow indicates the direction of increasing $F_{p}$ ). In Fig. 3(a) we can see that the threshold charge concentration decreases with $F_{\mathrm{p}}$, which means that we do not need a very high population inversion to reach the laser oscillation, and as a result, the laser oscillation condition is less restrictive. In Figs. 3(b) and (c) we can see that both the phonon and photon densities grow rapidly after the threshold, which is a clear signature of the costimulated emission of photons and phonons. But we can also observe that the phonon and photon densities do not grow in the same way. This can be explained by considering that the Purcell effect affects only the photons lifetime but not the phonons lifetime. This observation leaves an open door to investigate a possible Purcell effect for phonons. 


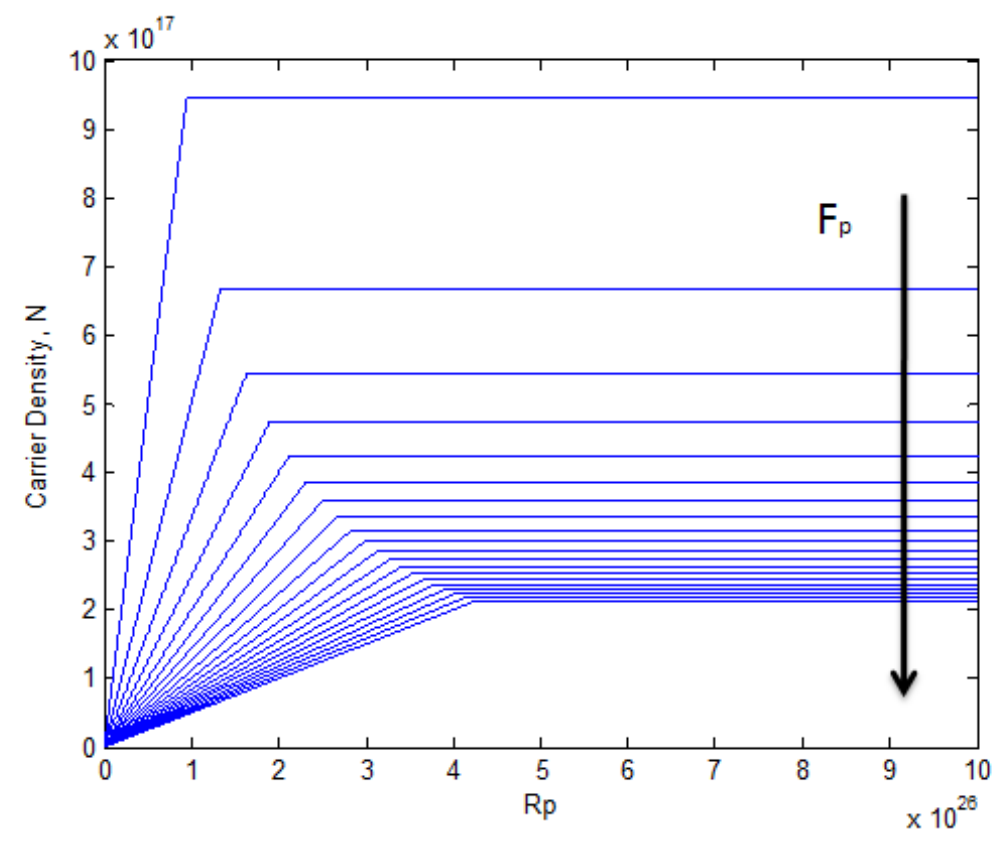

(a)

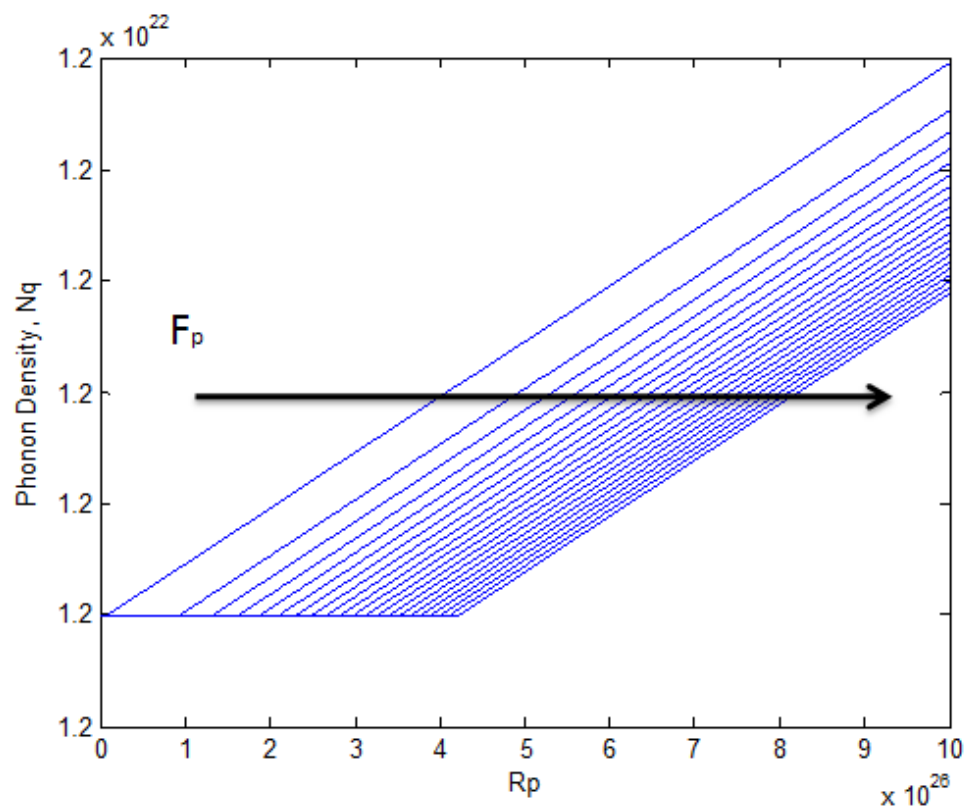

(b) 


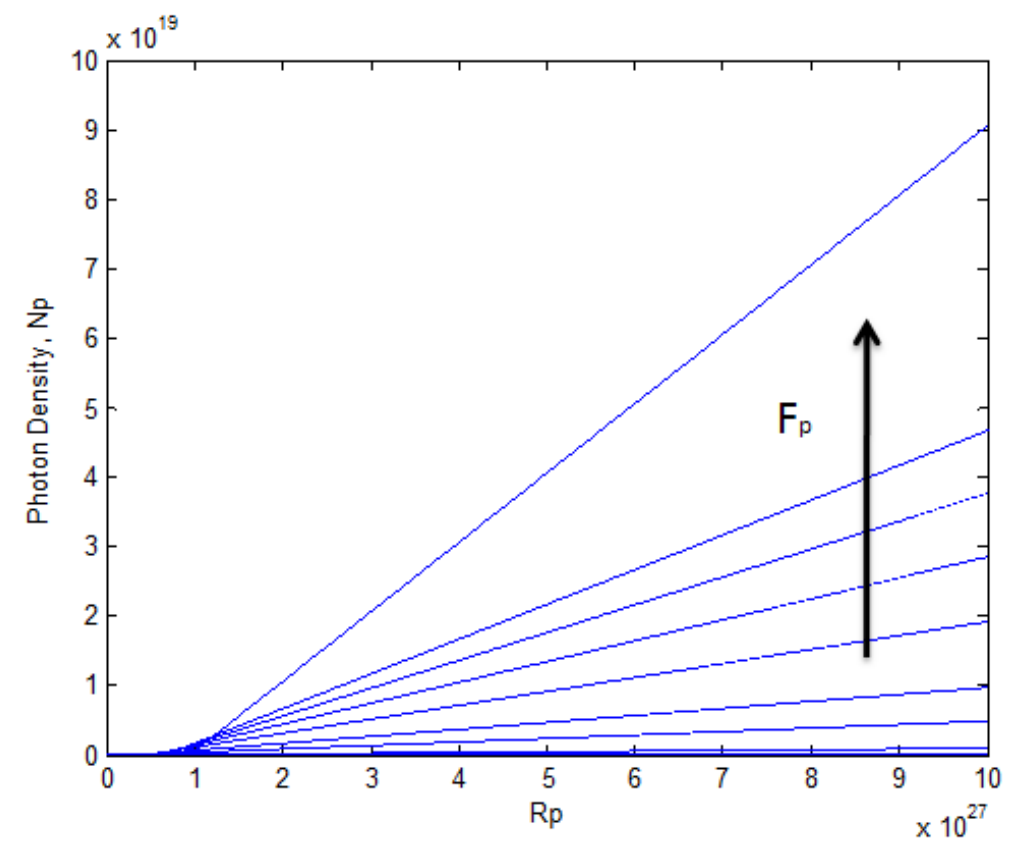

(c)

Figure 3. Carrier density (a), phonon density (b) and photon density (c) as a function of the pumping rate for values of the Purcell factor between 100 and 2000.

\section{Free-carrier absorption and optical gain}

In order to achieve optical amplification, and eventually lasing, the magnitude of optical amplification has to be large enough to overcome the optical losses resulting from the silicon itself and the optical cavity. A major loss mechanism that can hinder amplification is free carrier absorption (FCA). The FCA magnitude, $\alpha_{\mathrm{FC}}$, in bulk silicon at around room temperature is given by following empirical expression [25, 26],

$$
\alpha_{F C}=\left(1.01 \cdot 10^{-20} N+0.51 \cdot 10^{-20} P\right) \lambda^{2} T
$$

where $N$ and $P$ are, respectively, the electrons and holes densities. The expression of the optical gain inside the cavity as a function of $N$ is:

$$
g_{c a v}(\hbar \omega)=F_{P} \frac{h^{3} c^{2}}{8 \pi n^{2}(\hbar \omega)^{2}} M_{B}\left(n_{q}+1\right)\left[1-\frac{n_{q}}{n_{q}+1} \exp \left(\frac{(\hbar \omega+\hbar \Omega-\Delta F)}{K_{B} T}\right)\right] N^{2}
$$

If we compare the FCA (Eq. (38)) and the optical gain (Eq. (39)) for different values of Purcell factor we get that the optical gain the gain exceeds the FCA for $F_{p}>30$. However, this result is in contrast with the fact that optical gain in silicon cavities at room temperature has not been observed experimentally, which leads us to conclude that we need to consider also how the photonic cavity affects the FCA losses. In Ref. [27], T. F. Bogges and co-workers study both two photon absorption (TPA) and FCA in 
crystalline silicon. They describe the propagation of a optical pulse travelling along the $z$ direction taking into account the presence of linear absorption, TPA and FCA, using this expression:

$$
\frac{d I}{d z}=-\alpha I-\beta I^{2}-\sigma N I
$$

where $I$ is the irradiance, $\alpha$ is the linear absorption, $\beta$ is TPA coefficient and $\sigma$ is the FCA cross section. The irradiance dimension is: $[I]=W m^{2}=\frac{\Pi_{p} \hbar \omega}{t^{2}}$, where $\Pi_{\mathrm{p}}$ is the photon number. Since $\Pi_{p}=N_{p} V$ Eq. (40) can be transformed into:

$$
\frac{d \Pi_{p}}{d z}=-\alpha \Pi_{p}-\beta \Pi_{p}{ }^{2}-\sigma N \Pi_{p}
$$

And finally we get

$$
V \frac{d N_{p}}{d z}=-\alpha N_{p} V-\beta N_{p}^{2} V^{2}-\sigma N N_{p} \mathrm{~V}
$$

The last term in the right side of Eq.(42) is the loss due to FCA, $\alpha_{F C A}=\sigma N N_{p} \mathrm{~V}$. We obtained before that the photon density is proportional to Purcell factor inside of the cavity. Therefore, it is straightforward to conclude that the FCA losses inside of the cavity are proportional to $F_{\mathrm{p}}: \alpha_{F C A, c a v} \propto F_{P}$. The result is that both FCA losses and optical gain scale with the Purcell factor in the same way, just as it occurs in a bulk semiconductor. In the cavity at room temperature we get the results depicted in Fig. 4 which show an identical behaviour to those presented in Ref. [6] for bulk silicon.

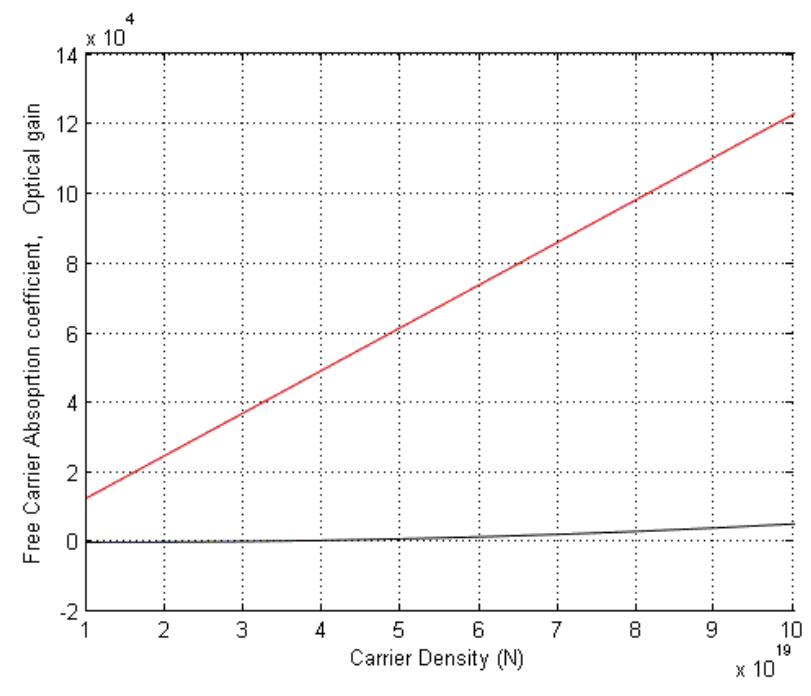

Figure 4. FCA loss (red line) and optical (black line) in an indirect bandgap semiconductor cavity at ambient temperature 
By taking into account this finding, we believe that the luminescence peaks from silicon cavities reported in Refs. [7,18-20] are not due to optical gain, but to an increase of the quantum efficiency in the process of emission. The quantum efficiency in the radiative process can be is defined as:

$$
\eta=\frac{W_{c a v}^{r a d}}{W_{c a v}^{r a d}+W^{n r a d}}
$$

Since $W_{\text {cav }}^{\text {rad }} \propto F_{P}$, we get:

$$
\eta=\frac{W_{b u l k}^{\text {rad }}}{W_{b u l k}^{\text {rad }}+\frac{W^{\text {nrad }}}{F_{P}}}
$$

It can be seen that the quantum efficiency approaches unity for large values of the Purcell factor, which can explain the luminescence peaks, but lasing is not feasible in silicon cavities at room temperature.

\section{Conclusion}

In this work we have described theoretically the different processes related to light emission from indirect bandgap semiconductor cavities. We have obtained that net optical gain in silicon at room temperature is not feasible despite the use of a high-Q photonic cavity since the Purcell factor affects the optical gain and the free-carrier absorption losses in the same way. In this sense, it has to be mentioned that we only have considered the losses to due free carrier absorption. However, other losses mechanisms will also co-exists in the system under study, which will further hinder the possibility of lasing emission.

\section{Acknowledgments}

This research has received funding from the European Community's Seventh Framework Programme (FP7/2007-2013) under grant agreement number 233883 (TAILPHOX). The authors wish to thank M. J. Chen for his useful comments.

\section{References}


[1] G. T. Reed and A. P. Knights, Silicon Photonics: An Introduction, John Wiley, West Sussex, 2004.

[2] L. Pavesi and D. J. Lockwood, Silicon Photonics, Springer-verlag, New York, 2004.

[3] M. Lipson, "Guiding, Modulating and Emitting Light on SiliconChallenges and Opportunities," J. Lightwave Technol. 23, 4222 (2005).

[4] W. P. Dumke, "Interband Transitions and Maser Action," Physical Review, vol. 127, pp. 1559-1563 (1962)

[5] T. Trupke, M. A. Green, and P. Wurfel, "Optical gain in materials with indirect transitions," Journal of Applied Physics, vol. 93, no. 11, pp. 9058-9061 (2003)

[6] M. J. Chen et al., "Optical Gain and Co-Stimulated Emissions of Photons and Phonons in Indirect Bandgap Semiconductors", Japanese Journal of Applied Physics, Vol. 45, No. 8B, pp. 6576-6588 (2006).

[7] L. Pavesi, L.dal Negro, C. Mazzoleni, G. Franz \& Ograve and F. Priolo, "Optical gain in silicon nanocrystals", Nature 408, 440-444, 23 November (2000).

[8] M. J. Chen, J. L. Yen, J. Y. Li, J. F. Chang, S. C. Tsai et al., "Stimulated emission in nanostructured silicon pn junction diode using current injection", Appl. Phys. Lett. 84, 2163 (2004).

[9] Sylvain G. Cloutier, Pavel A. Kossyrev and Jimmy Xu, "Optical gain and stimulated emission in periodic nanopatterned crystalline silicon", Nature Materials 4, 887 - 891 (2005).

[10] S. Iwamoto and Y. Arakawa, "Observation of enhacement photoluminescence from silicon photonic crystals nanocavity at room temperature", Appl. Phys. Lett. 91, 211104 (2007)

[11] S. Lin, Y. Kobayashi, Y. Ishikawa and K. Wada, "Luminescence enhancement by Si ring resonator structures on silicon on insulator", Appl. Phys. Lett. 92, 021113 (2008)

[12] M. Makarova, J. Vuckovic, H. Sanda and Y. Nishi,"Silicon-based photonic crystal nanocavity light emitters", Appl. Phys. Lett. 89, 221101-1 (2006)

[13] M. Fujita et al., "Light emission from silicon in photonic crystal nanocavity," IEEE J. Selected Topics Quantum Electron. 14, 1090 (2008). 
S. Iwamoto et al., "Observation of enhanced photoluminescence from silicon photonic crystal nanocavity at room temperature," Appl. Phys. Lett. 91, 211104 (2007).

[15] Shigeru Nakayama, Satomi Ishida, Satoshi Iwamoto, and Yasuhiko Arakawa, "Effect of cavity mode volume on photoluminescence from silicon photonic crystal nanocavities," Appl. Phys. Lett. 98, 171102 (2011).

[16] T. Trupke et al., "Very efficient light emission from bulk crystalline silicon", Appl. Phys. Lett. 82, 2996 (2003)

[17] E. M. Purcell, "Spontaneous emission probabilities at radio frequencies," Phys. Rev. 69, 681 (1946)

[18] Dieter K. Schroder, "Carrier Life Time in Silicon", IEEE Trans. Elec. Dev. Vol. 44, No. I, January 1997.

[19] L. Pavesi, "Silicon-Based Light sources for silicon Integrated Circuits", Advances in Optical Technologies, Vol. 2008 Art. ID 416926

[20] T. Trupke, M.A. Green, P. Würfel, P.P. Altermatt, A. Wang, J. Zhao and $\mathrm{R}$. Corkish, "Temperature dependence of the radiative recombination coefficient in intrinsic crystalline silicon", J. Appl. Phys. Vol. 94 No. 8 (2003)

[21] Vilson R.Almeda, Carlo A. Barrlos, Roberto R. Panepucii \& Michael Lipson. "All-optical control of light on a silicon chip", Nature (London) 431, 28 (2004)

[22] Charles Kittel, "Introduction to Solid State Physics", 4th Edition

[23] Bong-Shik Song, S. Noda, t. Asano and Y. Akahane, "Ultra-high-Q photonic double-heterostructure nanocavity", Nature Materials Vol. 4, March 2005.

[24] Y. Zhang and M. Loncar, "Ultra-high quality factor optical resonators based on semiconductor nanowires", Opt. Express 17400, Vol. 16 No. 22

[25] A. R. M. Zain, N. P. Johnson, M. Sorel and Richard M. De La Rue, "Ultra high quality factor one dimensional photonic crystal/photonic wire micro-cavities in silicon-on-insulator", Opt. Express 12084, Vol.16 No. 16

[26] J.C. Sturm and C.M. Reaves, "Silicon temperature measurement by infrared absorption: fundamental processes and doping effects", IEEE Trans. Elec. Dev. TED-39, pp. 81-88 (1992). 
[27] K. G. Svantesson and N. G. Nilsson, "Determination of the temperature dependence of the free carrier and interband absorption in silicon at $1.06 \mu \mathrm{m}$ " J. Phys. C 12, 3837 (1977).

[28] T. F. Boggess, J. R. Klaus, M. Bohnert, K. Mansour, S. C. Moss, I. W. Boyd and A. L. Smirl. "Simultaneous Measurement of the Two-Photon Coefficient and Free-Carrier Cross Section Above the Bandgap of Crystalline Silicon, IEEE J. of Quantum Electronics, Vol. QE. 22, No. 2, February 1986 